NASA/TM-2000-210225

\title{
Aeroacoustics Computation for Nearly Fully Expanded Supersonic Jets Using the CE/SE Method
}

Ching Y. Loh

Taitech Inc., Beavercreek, Ohio

Lennart S. Hultgren

Glenn Research Center, Cleveland, Ohio

Xiao Y. Wang

Taitech Inc., Beavercreek, Ohio

Sin-Chung Chang and Philip C.E. Jorgenson

Glenn Research Center, Cleveland, Ohio 
Since its founding, NASA has been dedicated to the advancement of aeronautics and space science. The NASA Scientific and Technical Information (STI) Program Office plays a key part in helping NASA maintain this important role.

The NASA STI Program Office is operated by Langley Research Center, the Lead Center for NASA's scientific and technical information. The NASA STI Program Office provides access to the NASA STI Database, the largest collection of aeronautical and space science STI in the world. The Program Office is also NASA's institutional mechanism for disseminating the results of its research and development activities. These results are published by NASA in the NASA STI Report Series, which includes the following report types:

- TECHNICAL PUBLICATION. Reports of completed research or a major significant phase of research that present the results of NASA programs and include extensive data or theoretical analysis. Includes compilations of significant scientific and technical data and information deemed to be of continuing reference value. NASA's counterpart of peerreviewed formal professional papers but has less stringent limitations on manuscript length and extent of graphic presentations.

- TECHNICAL MEMORANDUM. Scientific and technical findings that are preliminary or of specialized interest, e.g., quick release reports, working papers, and bibliographies that contain minimal annotation. Does not contain extensive analysis.

- CONTRACTOR REPORT. Scientific and technical findings by NASA-sponsored contractors and grantees.
- CONFERENCE PUBLICATION. Collected papers from scientific and technical conferences, symposia, seminars, or other meetings sponsored or cosponsored by NASA.

- SPECIAL PUBLICATION. Scientific, technical, or historical information from NASA programs, projects, and missions, often concerned with subjects having substantial public interest.

- TECHNICAL TRANSLATION. Englishlanguage translations of foreign scientific and technical material pertinent to NASA's mission.

Specialized services that complement the STI Program Office's diverse offerings include creating custom thesauri, building customized data bases, organizing and publishing research results ... even providing videos.

For more information about the NASA STI Program Office, see the following:

- Access the NASA STI Program Home Page at http://wwzw.sti.nasa.gov

- E-mail your question via the Internet to help@sti.nasa.gov

- Fax your question to the NASA Access Help Desk at (301) 621-0134

- Telephone the NASA Access Help Desk at (301) 621-0390

- Write to: NASA Access Help Desk NASA Center for AeroSpace Information 7121 Standard Drive Hanover, MD 21076 
NASA/TM-2000-210225

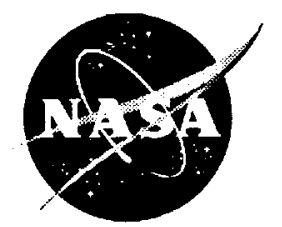

\section{Aeroacoustics Computation for Nearly Fully Expanded Supersonic Jets Using the CE/SE Method}

Ching Y. Loh

Taitech Inc., Beavercreek, Ohio

Lennart S. Hultgren

Glenn Research Center, Clevéeland, Ohio

Xiao Y. Wang

Taitech Inc., Beavercreek, Ohio

Sin-Chung Chang and Philip C.E. Jorgenson

Glenn Research Center, Cleveland, Ohio

Prepared for the

Sixth Aeroacoustics Conference

cosponsored by the American Institute of Aeronautics and Astronautics and the Confederation of European Aerospace Societies

Maui, Hawaii, June 12-14, 2000

National Aeronautics and

Space Administration

Glenn Research Center 
This report is a preprint of a paper intended for presentation at a conference. Because of changes that may be made before formal publication, this preprint is made available with the understanding that it will not be cited or reproduced without the permission of the author.

Available from

NASA Center for Aerospace Information

7121 Standard Drive

Hanover, MD 21076

Price Code: A03
National Technical Information Service 5285 Port Royal Road Springfield, VA 22100

Price Code: A03 
AIAA-2000-2010

\title{
AEROACOUSTICS COMPUTATION FOR NEARLY FULLY EXPANDED SUPERSONIC JETS USING THE CE/SE METHOD
}

\author{
Ching Y. Loh; Lennart S. Hultgren; Xiao Y. Wang;, \\ Sin-Chung Chang, and Philip C. E. Jorgenson \\ National Aeronautics and Space Administration \\ Glenn Research Center at Lewis Field \\ Cleveland, Ohio 44135, USA
}

\begin{abstract}
In this paper, the space-time conservation element solution element (CE/SE) method [1] is tested in the classical axisymmetric jet instability problem, rendering good agreement with the linear theory. The CE/SE method is then applied to numerical simulations of several nearly fully expanded axisymmetric jet flows and their noise fields and qualitative agreement with available experimental and theoretical results is demonstrated.
\end{abstract}

\section{Introduction}

Jet noise is a challenging topic in computational aeroacoustics. Generally, the computational scheme is required on the one hand to resolve the acoustic waves without introducing too much dispersion and dissipation, while on the other hand, it is required to capture shocks, or other nonlinear phenomena, near or inside the jet correctly. In addition, non-reflecting boundary conditions must be implemented in an efficient way either in the near field or in the far field.

The recent 'Space-Time Conservation Element and Solution Element Method' [2], or the CE/SE method in short, is a scheme that meets the above requirements. The CE/SE scheme possesses attractive properties for aeroacoustics computations in that: (i) it possesses low dispersion and dissipation errors; (ii) its shock-capturing nature makes the computation of shock-cell structures simple and accurate; (iii) the non-reflecting boundary conditions are simple and effective and can be applied in the near field of the jet without introducing excessive errors; and (iv) the vorticity, which plays an important

\footnotetext{
Taitech Inc., Member AIAA

tAssociate Fellow ALAA

Member AIAA
}

Copyright $\odot 2000$ by the American Institute of Aeronautics and Astronautics, Inc. No copyright is asserted in the United States under Title 17, U.S. Code. The U.S. Government has a royalty-free license to exercise all rights under the copyright claimed herein for government purposes. All other rights are reserved by the copyright owner.

NASA/TM-2000-210225 role in noise generating mechanisms, is determined without formal loss of accuracy. A detailed description of the CE/SE method can be found in the reports of Chang et al [3,4]. As demonstrated in our previous papers [5-8], the $\mathrm{CE} / \mathrm{SE}$ scheme is well suited for computing waves in compressible shear flows [5], vorticity/shock interaction [5-7], as well as the near-field noise of an underexpanded axisymmetric supersonic jet with a shock cell structure [7] - all of the examples being corner stones of the jetnoise phenomena.

In this paper, another aspect of jet aeroacoustics-the nearly fully expanded axisymmetric supersonic jet is investigated numerically by using the CE/SE Euler solver. The paper is arranged as follows: The axisymmetric CE/SE scheme is briefly discussed in Section 2. Section 3 considers the classical jet shear layer instability problem and numerical results are compared to those obtained by linear normal-mode instability theory. In Section 4 , the numerical results for several different types of noise fields of nearly fully expanded supersonic axisymmetric jets are presented and compared to experimental findings [10-12]. Conclusions are drawn in Section 5.

\section{Axisymmetric CE/SE Euler Solver}

In general, the CE/SE method systematically solves a set of integral equations derived directly from the physical conservation laws. It is distinguished by the simplicity of its conceptual basis-flux conservation in both space and time. Because of its integral form of the physical conservation laws, the scheme naturally captures shocks and other discontinuities in the flow. Both dependent variables and their derivatives are solved for simultaneously and, consequently, the flow vorticity can be obtained without reduction in accuracy. Non-reflective boundary conditions are also easily implemented because of the flux-conservation formulation.

\subsection{Conservation Form of the Unsteady Axisymmetric Euler Equations}

Consider a dimensionless conservation form of the unsteady axisymmetric Euler equations of a perfect gas. Let $\rho, u, v, p$, and $\gamma$ be the density, streamwise velocity component, radial velocity component, static pressure, and 1

American Institute of Aeronautics and Astronautics 
constant specific heat ratio, respectively. The axisymmetric Euler equations then can be written in the following vector form:

$$
U_{t}+F_{x}+G_{y}=Q,
$$

where $x, y \geqslant 0$, and $t$ are the streamwise and radial coordinates and time, respectively, and the conservative flow variable vector $U$ and the flux vectors in the streamwise and radial directions, $\boldsymbol{F}$ and $\boldsymbol{G}$, are given by:

$$
\boldsymbol{U}=\left(\begin{array}{c}
U_{1} \\
U_{2} \\
U_{3} \\
U_{4}
\end{array}\right), \quad \boldsymbol{F}=\left(\begin{array}{c}
F_{1} \\
F_{2} \\
F_{3} \\
F_{4}
\end{array}\right), \quad \boldsymbol{G}=\left(\begin{array}{c}
G_{1} \\
G_{2} \\
G_{3} \\
G_{4}
\end{array}\right),
$$

where $U_{m}, F_{m}, G_{m}, m=1,2,3,4$ are the same as in the standard 2-D CE/SE formulation [3, 4] and

$$
\boldsymbol{Q}=\left(\begin{array}{l}
Q_{1} \\
Q_{2} \\
Q_{3} \\
Q_{4}
\end{array}\right)
$$

with

$$
\begin{gathered}
Q_{1}=-U_{3} / y, \quad Q_{2}=-U_{2} U_{3} / U_{1} y \\
Q_{3}=-U_{3}^{2} / U_{1} y, \quad Q_{4}=-G_{4} / y .
\end{gathered}
$$

The axisymmetric Euler equations can be thought of in this formulation as a variation of their two-dimensional counterparts with 'source' terms on their right-hand sides. Of course, these additional terms do depend implicitly on the solution to the equations and, hence, are not true source terms, but they can formally be treated as such for the purpose of deriving the numerical scheme.

By considering $(x, y, t)$ as coordinates of a threedimensional Euclidean space $E_{3}$ and using Gauss divergence theorem, it follows that Eq. (1) is equivalent to the following integral conservation law:

$$
\oint_{S(V)} \boldsymbol{H}_{m} \cdot \mathrm{d} \mathbf{S}=\int_{V} Q_{m} \mathrm{~d} V, \quad m=1,2,3,4,
$$

where $S(V)$ denotes the surface around a volume $V$ in $E_{3}$ and $\boldsymbol{H}_{m}=\left(F_{m}, G_{m}, U_{m}\right)$.

\subsection{CE/SE Structure}

In the CE/SE scheme, the flux conservation relation in space-time is the only mechanism that transfers information between node points. The conservation element $C E$, or computational cell, is the finite volume to which the integral flux condition (2) is to be applied. Discontinuities are allowed to occur in the interior of a conservation element as long as the integrals on the right-hand side of (2) exist. A solution element $S E$ associated with a grid node is here a set of three interface planes in $E_{3}$ that passes through this node. The solution, i.e. $U, U_{x}$, NASA/TM-2000-210225 and $U_{y}$ are calculated at this node. Within a given solution element $S E(j, k, n)$, where $(j, k, n)$ is the node index, the flow variables are not only considered continuous but are also approximated by linear Taylor expansions. This procedure is identical to the one in the standard 2-D CE/SE Euler solver [3, 4].

The discrete approximation of (2) is then

$$
\oint_{S(C E)} H_{m}^{*} \cdot \mathrm{d} \mathbf{S}=V(C E) \sum_{(j, k, n) \in S(C E)} \beta_{j, k}^{n}\left(Q_{m}\right)_{j, k}^{n},
$$

for $m=1,2,3,4$, where $\boldsymbol{H}_{m}^{*}=\left(F_{m}^{*}, G_{m}^{*}, U_{m}^{*}\right)$ denotes the linear Taylor-series approximation of the corresponding 'unstarred' variables and $\sum_{(j, k, n) \in S(C E)} \beta_{j, k}^{n}=1$. The right-hand side of (3), in general, is the volume of the $C E$ times a weighted average of the 'source' term evaluated at the nodes on $S(C E)$. Each $S(C E)$ is made up by surface segments belonging to two neighboring $S E$ 's. All the unknowns are solved for based on these relations. No extrapolations (interpolations) across a stencil of cells are needed or allowed. In order that the num-

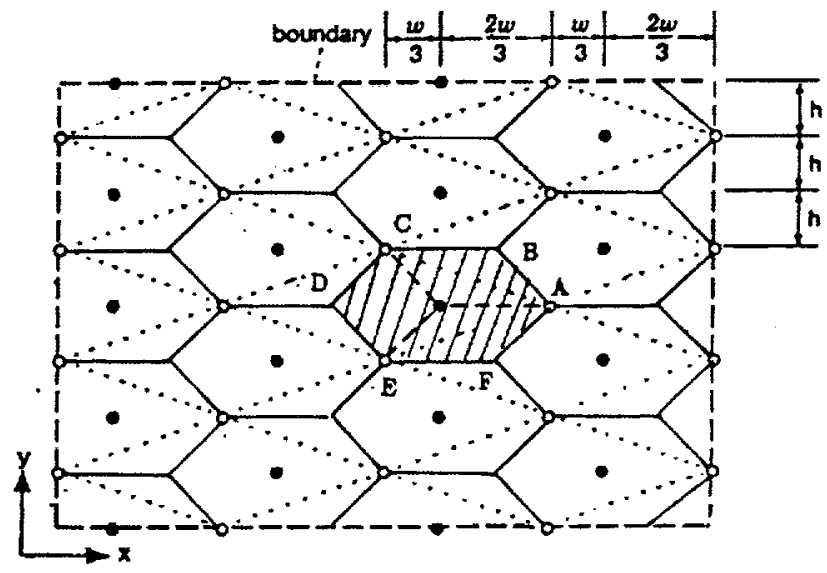

Figure 1: Double staggered grid in $E_{3}$.

ber of equations derived from the flux conservation law above matches the number of unknowns (here 12 scalar unknowns), the grid in $(x, y)$ plane needs to be carefully designed $[3,4]$. The mesh is staggered in both time and space. In a spatial plane in $E_{3}$, the grid nodes, see Fig. 1, are grouped as two staggered sets $\Omega_{1}$ (open circles) and $\Omega_{2}$ (filled circles). At a given time step the unknowns are evaluated only on one of the grid sets, $\Omega_{1}$ or $\Omega_{2}$, and at the next it will be evaluated on the other set, i.e., the spatial sets alternate as time is stepped forward. As can be seen in this figure, each 'interior' node point then has three nearest neighbors at the previous time step. Thus, there are three $C E$ 's associated with each node point in this arrangement and, therefore, there are the same number of relations as there are unknowns. 


\subsection{Treatment of the Source Term}

In solving (3), only the $\beta_{j, k}^{n}$ corresponding to the new (half) time level is taken to be nonzero (and equal to unity). This strategy has successfully been applied by Yu et al [9] to problems with very stiff source terms.

As the source term $Q=Q(U)$ itself is a function of the unknown $U$ at the new (half) time level, a local iterative procedure is needed to determine $U$. For our particular choice of $\beta_{j, k}^{n}$, the discretized integral equation (3) reduces to the form

$$
\mathbf{U}-\mathbf{Q}(\mathbf{U}) \frac{\Delta t}{2}=\mathbf{U}_{H},
$$

where $U_{H}$ is the local homogeneous solution $(Q=0$ locally). Note that $U_{H}$ is in fact the standard 2-D Euler solution at the previous (half) time step, obtained by explicit formulas. A Newton iterative procedure to determine $U$ is then

$$
\boldsymbol{U}^{(i+1)}=\boldsymbol{U}^{(i)}-\left(\frac{\partial \boldsymbol{\Phi}}{\partial \bar{U}}\right)^{-1}\left[\boldsymbol{\Phi}\left(\boldsymbol{U}^{(i)}\right)-\boldsymbol{U}_{H}\right],
$$

where $i$ is the number of iteration and

$$
\boldsymbol{\Phi}(\boldsymbol{U})=\boldsymbol{U}-\boldsymbol{Q}(\boldsymbol{U}) \frac{\Delta t}{2} .
$$

Normally, $U$ at the previous (full) time step is a good initial guess $U^{(0)}$ and the procedure takes about 2-3 iterations to convergence. The Jacobian matrix is given by

$$
\frac{\partial \Phi}{\partial \boldsymbol{U}}=\left(\begin{array}{cccc}
1 & 0 & \frac{\Delta t}{2 y} & 0 \\
-\frac{U_{2} U_{3} \Delta t}{2 U^{2} y} & 1+\frac{U_{3}^{2} \Delta t}{2 U_{1} y} & \frac{U_{2} \Delta t}{2 U_{1}^{2} y} & 0 \\
-\frac{U_{3}^{2} \Delta t}{2 U_{1}^{2} y} & 0 & 1+\frac{U_{3} \Delta t}{2 U_{1} y} & 0 \\
A_{1} & A_{2} & A_{3} & 1+\frac{\gamma U_{3} \Delta t}{4 U_{1} y}
\end{array}\right)
$$

where

$$
\begin{gathered}
A_{1}=-\frac{U_{3} \Delta t}{2 U_{1} y}\left[\gamma U_{4}-\frac{(\gamma-1)\left(U_{2}^{2}+U_{3}^{2}\right)}{U_{1}}\right] \\
A_{2}=-(\gamma-1) \frac{U_{2} U_{3} \Delta t}{2 U_{1}^{2} y} \\
A_{3}=\frac{\Delta t}{2 y}\left[-\frac{\gamma-1}{2} \frac{U_{2}^{2}+3 U_{3}^{2}}{U_{1}^{2}}+\frac{\gamma U_{4}}{U_{1}}\right] .
\end{gathered}
$$

The inverse of the Jacobian, i.e., $\left(\frac{\partial \Phi}{\partial U}\right)^{-1}$ can easily be derived analytically for this particular case, thus, leading to savings in CPU time.

\section{Free Jet Shear Layer Instability}

In the first example, we study the inviscid linear and nonlinear instability properties of an axisymmetric free shear layer (jet). This important class of flows forms the basis of jet noise generation theory and have been NASA/TM-2000-210225 the subject of many detailed analytical and experimental studies.

The direct numerical computation of the shear-layer instability, using the CE/SE Euler scheme, is compared with linear results obtained using the normal mode approach. The background mean flow consists of a fast jet stream and a slow ambient stream around it, with the two parallel streams connected by a continuously changing annular shear layer. The streamwise flow variables in the fast jet stream are taken as the corresponding scales for the nondimensionalization. The length scale is taken as $\delta / 2$, where $\delta$ is the vorticity thickness and is defined as

$$
\delta=\left(U_{1 *}-U_{2 *}\right) /\left(\mathbf{d} U_{*} / \mathbf{d} y_{*}\right)_{\max }
$$

with the subscript ' $*$ ' here denoting dimensional quantities. In a parallel flow, the mean pressure is constant and the mean transverse velocity vanishes all over the domain, while the mean streamwise velocity and density profiles must be specified.

In the linear stability theory, the normal-mode assumption is made, i.e.,

$$
\phi(x, y, t)=\hat{\phi}(y) \exp [\mathrm{i}(\alpha x-\omega t)],
$$

where $\phi(x, y, t)$ denotes the perturbation streamwise and radial velocities $u^{\prime}, v^{\prime}$, pressure $p^{\prime}$. or density $\rho^{\prime}$; and $\alpha$, $\omega$ are the complex wave number and real angular frequency of the disturbance, respectively. We note that $\hat{u}$, $\hat{v}, \hat{p}$ and $\hat{\rho}$ are also complex functions in general. Adding the above perturbations to the corresponding mean flow variables and substituting them into the $3-\mathrm{D}$ axisymmetric Euler equations, a set of equations for the unknown perturbation flow variables is obtained. Assuming that the mean flow variables satisfy the Euler equations and by eliminating those terms of second order or higher, the compressible Rayleigh equation in terms of the perturbation pressure eigenfunction component $\hat{p}$ can be achieved after some manipulation.

To be specific, the streamwise mean flow velocity $U$ is here taken as

$$
U(y)=\frac{1+R \tanh y}{1+R},
$$

where $R$ is the velocity ratio of the shear layer. We further assume for simplicity that the Prandtl number is unity and the mean flow temperature $\mathrm{T}$ distribution is then obtained from the Buseman-Crocco relation:

$T=T_{2}+\frac{1-T_{2}}{1-U_{2}}\left(U-U_{2}\right)+\frac{1}{2}(\gamma-1) M I_{1}^{2}\left(U-U_{2}\right)(1-U)$,

where the subscripts 1 and 2 denote the fast stream and slow stream variables respectively. $T$ in turn yields the density distribution across the shear layer. As in [6], we choose the parameters

$$
R=0.15, \quad M_{1}=1.5, \quad T_{2}=1.85
$$


Then, the nondimensional flow states are worked out as:

$$
\begin{gathered}
U_{1}=1, \quad V_{1}=0, \quad p_{1}=1 / 3.15 \\
T_{1}=1, \quad \rho_{1}=1, \quad M_{1}=1.5 \\
U_{2}=.7391304, \quad V_{2}=0, \quad p_{2}=1 / 3.15 \\
T_{2}=1.85, \quad \rho_{2}=0.5405405 .
\end{gathered}
$$

Under the above conditions, with an appropriate $\omega$ chosen, the complex eigenfunction component $\hat{p}=\hat{p}_{r}+\mathrm{i} \hat{p}_{i}$ and the corresponding eigenvalue $\alpha=\alpha_{r}+\mathrm{i} \alpha_{i}$, where the subscripts $r$ and $i$ denote real and imaginary parts respectively, can be found by solving the compressible Rayleigh equation.

\subsection{Linear, Nonlinear Instability of an Axisymmetric Jet and Vortex Roll-Up}

In studying the instability of a free jet shear layer and the consequent vortex roll-up and noise generation, one is mainly interested in the most unstable mode which dominates the nonlinear vortex roll-up. In order to investigate such instabilities, we shall enforce a small harmonic perturbation at a single frequency at the inlet boundary and compute the resulting development of the spatial instability wave. In this test example, the computational domain spans between $0 \leqslant x \leqslant 300$ and $0 \leqslant y \leqslant 20$, with a grid of $601 \times 101$, respectively (i.e. $\Delta x=0.5$ and $\Delta y=0.2$ ). A time step size $\Delta t=0.15, \epsilon=0.5$ and the weighted average index $\alpha=0$ were found appropriate. The computation of the unsteady jet flow is carried out until $t=390$ ensuring that the spatial instability is fully developed.

From a chart of $\omega$ versus $-\alpha_{i}$, for the case under consideration here, it can be observed that the exponential growth rate $-\alpha_{i}$ reaches its maximum of about 0.0244 when the angular frequency $\omega=0.405$ and the latter is the value used in the following test example. The complex spatial eigenvalue $\alpha=0.44726-0.02444 \mathrm{i}$ and the corresponding eigenmode is then determined by solv. ing the compressible Rayleigh equation for this value of $\omega$. The eigenfunction is normalized such that the maximum $r . m . s$ value of the streamwise velocity component is unity. The forcing at the inlet boundary $(x=0)$ then has the form

$$
\begin{gathered}
\phi=\delta\left[\hat{\phi}_{r} \cos \omega t+\hat{\phi}_{i} \sin \omega t\right], \\
\frac{\partial \phi}{\partial x}=\delta\left[-\left(\hat{\phi}_{r} \alpha_{i}+\hat{\phi}_{i} \alpha_{r}\right) \cos \omega t-\left(\hat{\phi}_{i} \alpha_{i}-\hat{\phi}_{r} \alpha_{r}\right) \sin \omega t\right], \\
\frac{\partial \phi}{\partial y}=\delta\left[\hat{\phi}_{r}^{\prime} \cos \omega t+\hat{\phi}_{i}^{\prime} \sin \omega t\right],
\end{gathered}
$$

where $\phi$ denotes any of the eigenfunction components $u^{\prime}, v^{\prime}, p^{\prime}$, or $\rho^{\prime}$, and $\delta$ is the forcing amplitude. To fully demonstrate the capability of the CE/SE scheme to compute both lincar and nonlinear instability waves, the forcing amplitude $\delta$ is taken to be $1 / 1000$.

Fig. 2 displays the flow variables contours. Vortex roll-up is clearly observed in this figure. This figure clearly demonstrates the effectiveness of the nonreflecting boundary conditions at the top, bottom and outlet. We emphasize that the domain shown in the figures is exactly the computational domain, no buffer zones, cut-offs or other numerical fixes are applied.

To assess the grid dependence of the numerical results, the computation was repeated with half grid sizes and time step size. Fig. 3 shows that they are quite similar except that, as expected, the finer grid allows higher harmonics to be better resolved.

Fig. 4 shows the power spectral density PSD of the computed $p^{\prime}$ in $\log _{10}$ scale at the streamwise station $x=$ 150. The spectral peak at the forcing frequency $f=$ $\frac{w^{2}}{2 \pi}=0.06446$, the second, third, and fourth harmonic thereof are clearly displayed.

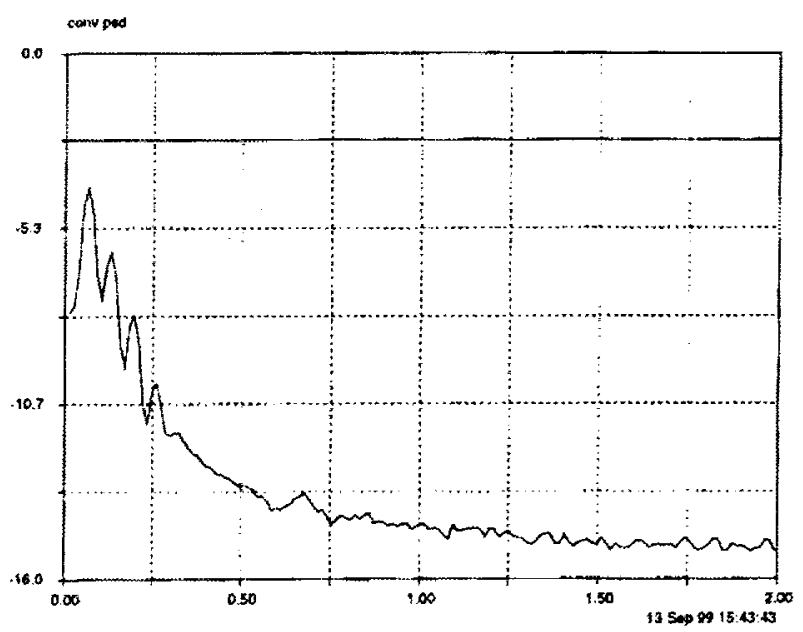

Figure 4: Power spectral density of the jet shear layer at $x=150$.

\section{Mach radiation of jets}

In Oertel's experiments [10-12] and Tam and Hu's analysis [13], three distinct types of Mach waves for high-speed jets were identified. Our purpose in this section is to investigate these three types of axisymmetric fully expanded circular jets. The numerical results are presented and qualitatively compared to experimental findings [10-12]. In all the examples, a perturbation (or forcing) is provided by a right-hand-side source term in the energy equation, the 4 th component of Eq. (1), located at the origin $(0,0)$ inside the jet core:

$$
\frac{\delta}{\gamma-1} \exp \left[-B\left(x^{2}+y^{2}\right)\right] \cos (\omega t)
$$


where $\delta$ is a small number $(0.00005<\delta<0.001)$, $\omega=2 \pi S t$, and the constant $B=8$. Even though the $C E / S E$ implementation of non-reflective boundary conditions is very effective (generally less than $1 \%$ reflection), it is of advantage to also add a (streamwise) buffer zone to the outflow side of the computational domain to further reduce numerical reflections from radial regions where the mean flow changes rapidly. A buffer zone of 20 cells with exponentially increasing size was used in the computations presented below and effectively kept the computed acoustic field free of such reflections. Due to the intrinsic properties of the $C E / S E$ scheme, it is likely that the number of buffer region cells could be substantially reduced without a significant degradation of the computed results - this is yet to be studied, however.

\subsection{Mach radiation from a supersonic axisymmetric jet}

In this test example, a supersonic jet with Mach number $M_{j}=2.0$ is considered. As the jet flow is fully expanded, the jet nozzle exit pressure is the same as the ambient pressure. However, tiny fluctuations of pressure are still allowed and accounts for the acoustic wave generation and propagation. The overall motion is approximately in an axisymmetric mode [14] at the Strouhal number $S t=0.2$. We note that since the Euler solution does not account for the shear layer spreading due to viscous effects, our numerical results are not expected to perfectly match the experimental [15] sound pressure level, SPL.

The computational domain spans between $0 \leqslant x \leqslant$ $33 D$ and $0 \leqslant y \leqslant 19 D$, with a non-uniform grid of $350 \times 281$ nodes, where $D$ is the diameter of the jet at the nozzle exit. More grid points are packed around the shear layer. The last 50 cells in $x$ direction grow exponentially in length and are used as buffer zone. The perturbation source strength $\delta=0.001$. A time step size $\Delta t=0.005, \epsilon=0.5$ and the weighted average index $\alpha=0$ are found appropriate. The computation of the unsteady jet flow is carried out until $t=100$ ensuring that the spatial instability is fully developed.

Fig. 5 illustrates the isobars and v-velocity contours in the near and intermediate field. The Mach radiation flow pattern agrees qualitatively with experimental [15] and other computational [14] results and is categorized by Oertel [10] as Type I Mach radiation.

\subsection{Mach radiation from a supersonic axisymmetric jet at low Strouhal number}

In this test, the same supersonic $M_{j}=2.0$ jet is considered but a low Strouhal number $S t=0.07$ is chosen. The computational domain spans between $0 \leqslant x \leqslant 8 D$ and $0 \leqslant y \leqslant 2.5 D$, with a non-uniform grid of $630 \times 151$ nodes. The last 30 cells in $x$ direction grow exponentially in length and are used as buffer zone. The per- turbation source strength $\delta=0.0001$. A time step size $\Delta t=0.002, \epsilon=0.5$ and the weighted average index $a=0$ are found appropriate. The computation of the unsteady jet flow is carried out until $t=27$.

Fig. 6 demonstrates the isobars in the near field. At a low Strouhal number, the radiated Mach waves are quite weak and rather steeply inclined. They look almost vertical. This is categorized as Type II Mach radiation by Oertel [11]. Tam and Hu [13] also give an explanation of the phenomena in their analysis. Inside the jet core, the cross-hatch pattern of a trapped Mach wave system is observed. As will be seen in the next subsection, such a phenomenon is more pronounced when $M_{j}$ is low.

\subsection{Mach wave system inside the jet}

This is the Type III experimental result by Oertel [12]. It was found that, as analyzed by Tam and $\mathrm{Hu}$ [13], the Mach radiation attenuates exponentially outside the jet shear layer, while the Mach wave system is trapped inside the jet and forms a cross-hatch pattern. Here the jet shear layer plays a role as a 'wave guide'. We consider two cases in this example.

4.3.1 Case 1: $M_{j}=1.2$ In the first case, a supersonic jet with low Mach number $M_{j}=1.2$ is considered. The computational domain is $0 \leqslant x \leqslant 3 D$ and $0 \leqslant y \leqslant 1 D$, with a non-uniform grid of $330 \times 101$ nodes, where again $D$ is the diameter of the jet at the nozzle exit. The last 30 cells in $x$ direction are used as buffer zone. Here, the near field is chosen for investigation since our attention is focussed on the Mach wave system inside the jet. The perturbation source strength $\delta=0.00005$, with a high Strouhal number $S t=2.0$. A time step size $\Delta t=0.0015, \epsilon=0.5$ and the weighted average index $\alpha=0$ are chosen. The computation of the unsteady jet flow is carried out for 12000 steps to ensure that the spatial instability is fully developed.

Fig 7 clearly demonstrates the cross-hatch pattern of the Mach wave system inside the 'wave guide'-the jet core. The Mach waves are repeatedly reflected because they do not penetrate the annular shear layer. The pattern qualitatively agrees with Oertel's experiment [12] (Type III) and the analysis of Tam and Hu [13]. At a low supersonic Mach number $M_{j}=1.2$, at the outer surface of the shear layer, the local phase velocity can hardly exceed the local speed of sound and hence practically no exterior Mach radiation occurs.

4.3.2 Case 2: $M_{j}=2.0 \quad$ In the second case, the Mach number $M_{j}$ is increased to 2.0. The computational domain is $0 \leqslant x \leqslant 7.5 D$ and $0 \leqslant y \leqslant 1 D$, with a nonuniform grid of $280 \times 101$ nodes. As before, the last 30 cells in $x$ direction are used as buffer zone. The perturbation source strength $\delta=0.001$, with the Strouhal number $S t=2.0$. A time step size $\Delta t=0.002, \epsilon=0.5$ 
and the weighted average index $\alpha=0$ are chosen. The computation of the unsteady jet flow is again carried out for 12000 steps.

Fig 8 displays the cross-hatch pattern of the Mach wave system inside the 'wave-guide' jet core. The pattern qualitatively agrees with the analysis of Tam and $\mathrm{Hu}$ [13]. At a supersonic Mach number $M_{j}=2$, the local phase velocity at the outer shear layer may exceed the local speed of sound and hence Mach radiation is generated.

\section{Concluding Remarks}

In this paper, the noise field of nearly fully expanded axisymmetric jets are investigated by using the recent CE/SE Euler scheme. For the classical jet-shear-layer instability problem, the numerical results show a linear initial development of the spatially growing instability wave (in good agreement with stability theory) followed by nonlinear effects leading to vortex roll up. Many aspects of the computed results for Mach radiation are also in good agreement with Oertel's experimental findings [10-12] and Tam's analysis [13].

The advantages of the scheme as claimed in Section 1 are confirmed in the present simulations. The implementation is "effortless" in that no special treatment and parameter selections are needed.

\section{References}

[1] Chang, S. C., "The Method of Space-Time Conservation Element and Solution Element-A New Approach for Solving the Navier-Stokes and Euler Equations," Journal of Computational Physics. vol. 119, pp. 295-324 (1995).

[2] Chang, S. C. and To, W. M., "A Brief Description of a New Numerical Framework for Solving Conservation Laws-The Method of Space-Time Conservation Element and Solution Element," Proceedings of the Thirteenth International Conference on $\mathrm{Nu}$ merical Methods in Fluid Dynamics, Rome, Italy, 1992, M. Napolitano and F. Sabetta, eds., Lecture Notes in Physics 414, Springer-Verlag, pp. 396400. Also published as NASA TM 105757 (1992).

[3] Chang, S. C., Yu, S. T., Himansu, A., Wang, X. Y., Chow, C. Y. and Loh, C. Y., "The Method of Space-Time Conservation Element and Solution Element-A New Paradigm for Numerical Solution of Conservation Laws", Computational Fluid Dynamics Review, eds. M. M. Hafez and K. Oshima, Wiley (1997).

[4] Chang, S. C., Wang, X. Y. and Chow, C. Y., "The Space-Time Conservation Element and Solution Element Method - A New High-Resolution and Gen- uine Multidimensional Paradigm for Solving Conservation Laws ", J. Comput. Phys. vol. 156, pp. 89-136 (1999).

[5] Loh, C. Y., Hultgren, L. S. and Chang, S.-C., "Computing Waves in Compressible Flow Using the Space-Time Conservation Element Solution Element Method," AIAA Paper 98-0369 (1998).

[6] Loh, C. Y., Hultgren, L. S., Chang, S.-C. and Jorgenson, P. C. E., "Vortex Dynamics Simulation in Aeroacoustics by the Space-Time Conservation Element Solution Element Method," AIAA Paper 990359 (1999).

[7] Loh, C. Y., Hultgren, L. S., Chang, S.-C. and Jorgenson, P. C. E., "Noise Computation for a ShockContaining Supersonic Axisymmetric Jet Using the CE/SE Method" AIAA Paper 2000-0475 (2000).

[8] Loh, C. Y., Chang, S. C. and Scott, J. R., "Computational Aeroacoustics via the Space-Time Conservation Element/Solution Element Method", AIAA Paper 96-1687 (1996).

[9] Yu, S. T. and Chang, S.-C., "Treatment of Stiff Source Terms in Conservation Laws by the Method of Space-Time Conservation Element and Solution Element", AIAA Paper 97-0435 (1997).

[10] Oertel, H., " Mach wave radiation of hot supersonic jets", in Mechanics of Sound Generation in Flows, (ed. E.A.Muller), pp. 275-281, Springer (1979).

[11] Oertel, H., " Mach wave radiation of hot supersonic jets investigated by means of the shock tube and new optical techniques", Proceedings of the 12th Intl. Symp. on Shock Tube and Waves, Jerusalem (eds. A. Lifshitz and J.Rom), pp. 266-275 (1980).

[12] Oertel, H., " Coherent structures producing Mach waves inside and outside of the supersonic jet ", Structure of Complex Turbulent Shear Flow, IUTAM Symp. Marseille (1982).

[13] Tam, C. K. W, and Hu, F. Q., "On the three families of instability waves of high-speed jets", J. Fluid Mech., vol. 201, pp. 447-483 (1989).

[14] Shih, S. H., Hixon, D. R. and Mankbadi, R. R., "Zonal Approach for Prediction of Jet Noise", AIAA J., vol.13, pp. 745-752 (1997).

[15] Troutt, T. R. and McLaughlin, D. K., " Experiments on the flow and acoustic properties of a moderateReynolds-number supersonic jet", J. Fluid Mech., vol. 116, pp. 123-156 (1982). 


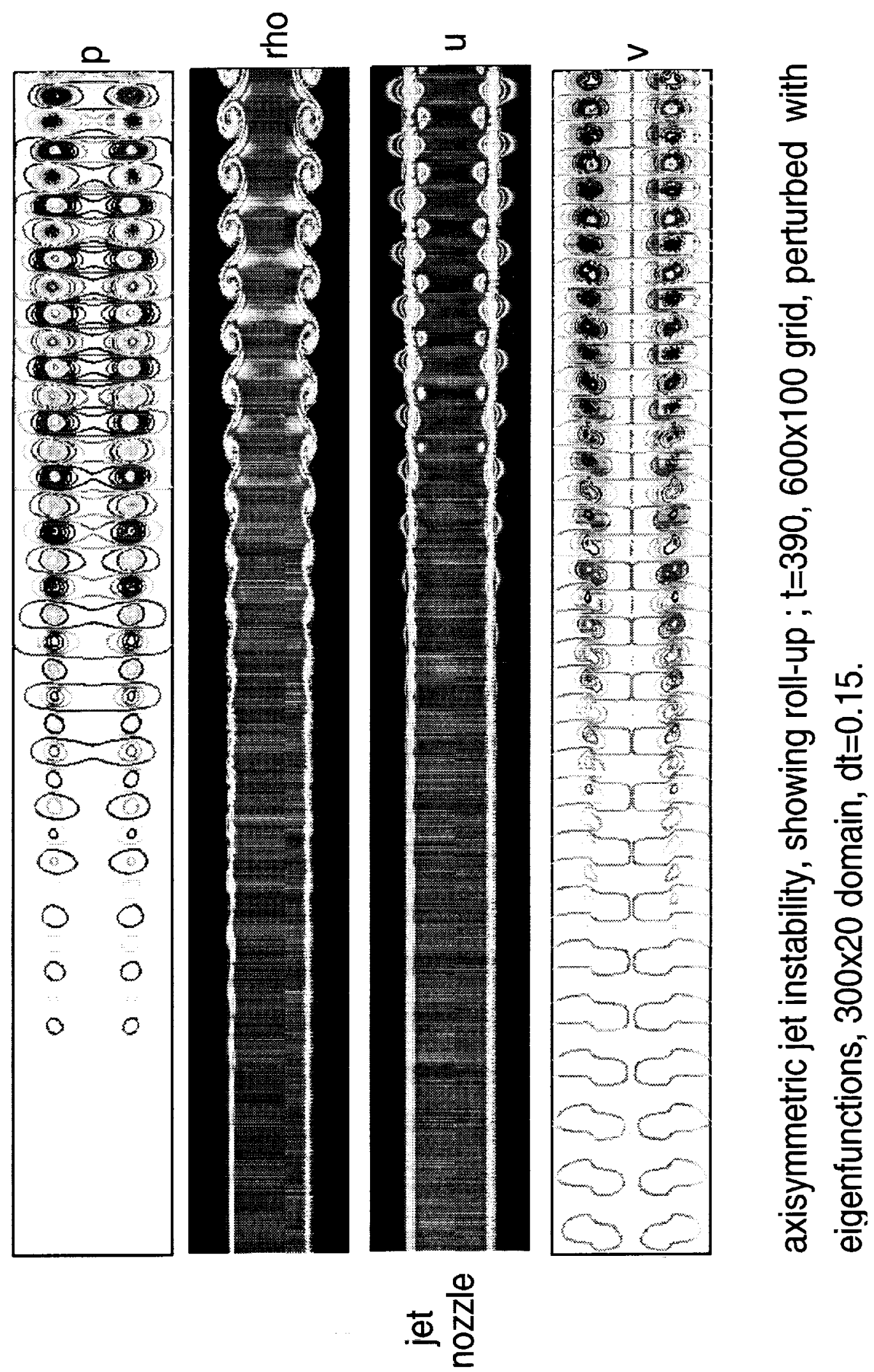

Figure 2: Contours for jet instability problem, with coarse grid at $t=390$. 

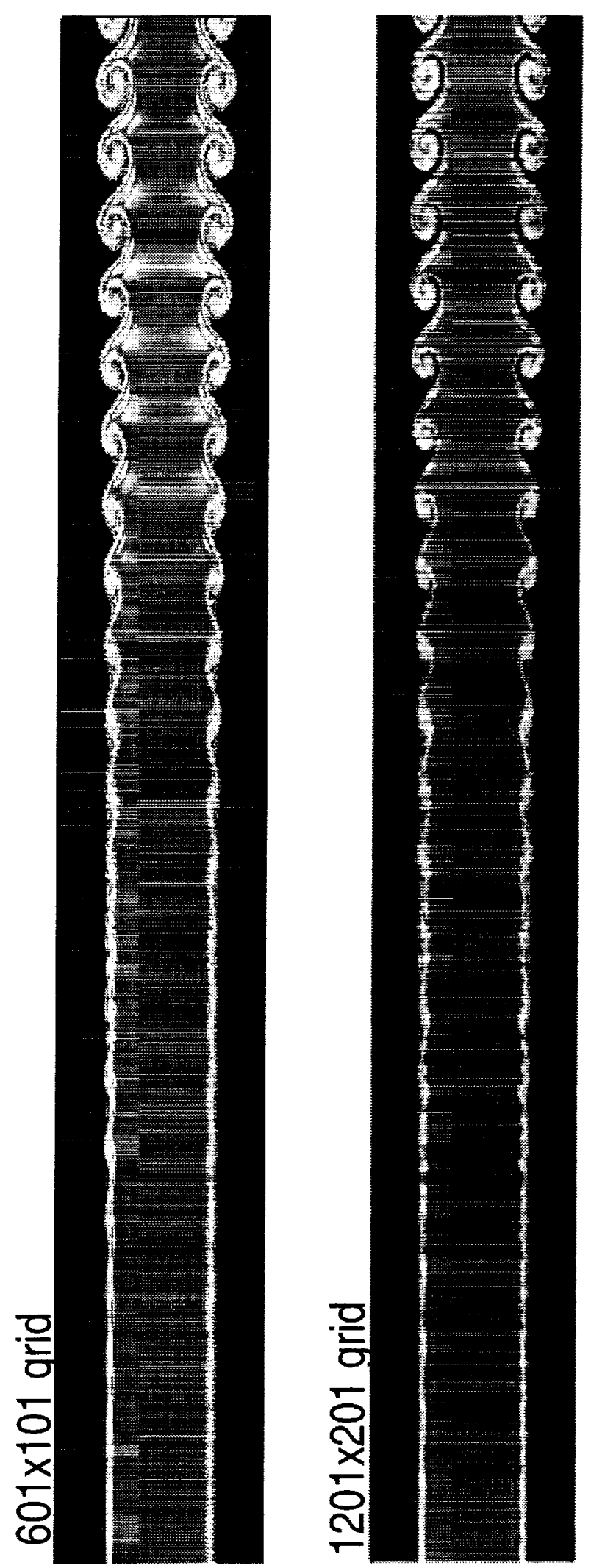

Figure 3: Comparison of density contours for coarse and fine grids at $t=390$. 

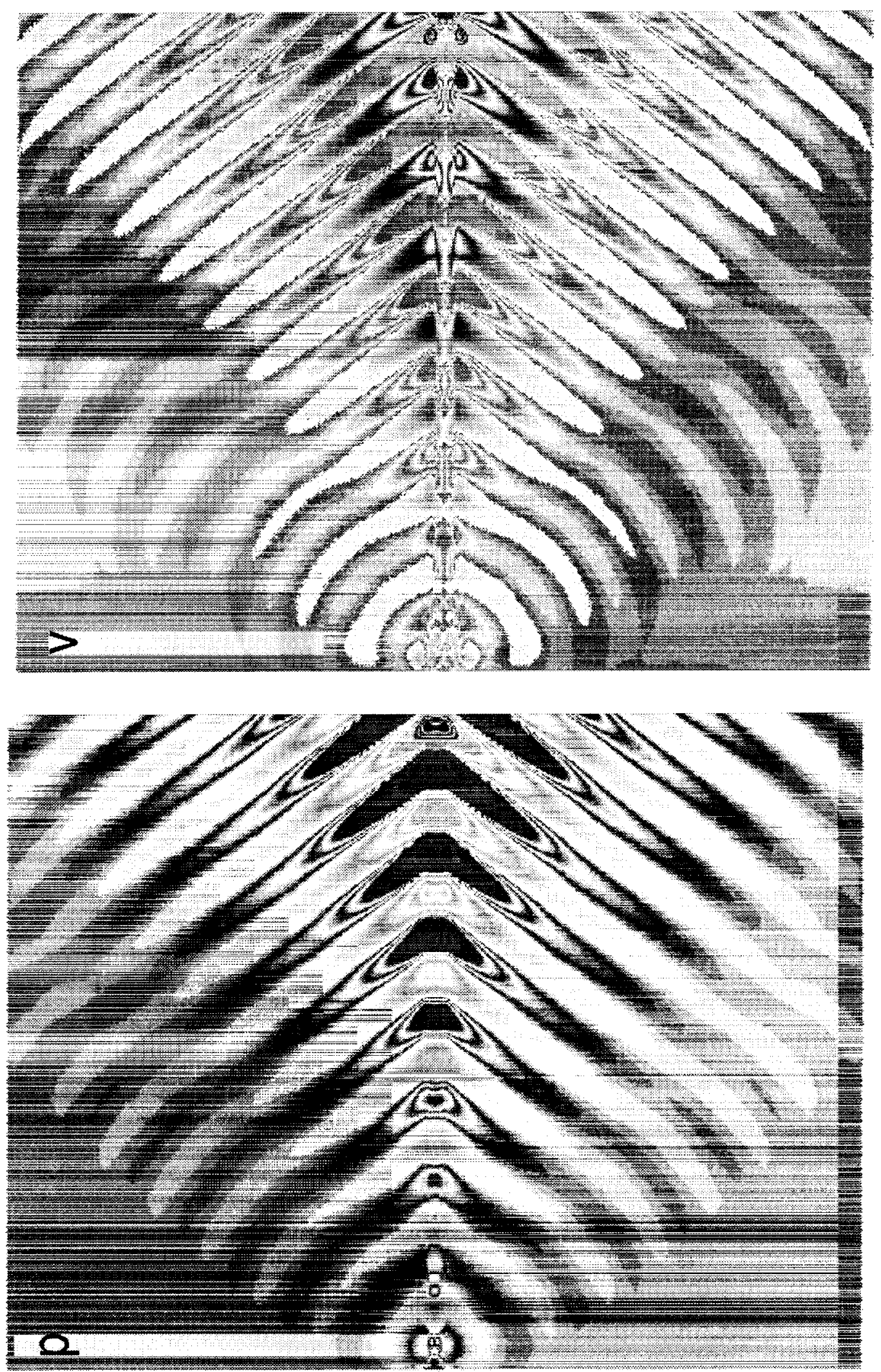

Figure 5: Type I Mach radiation of a supersonic jet at $M_{j}=2,0$ and Strouhal number $S t=0.2$, domain size 33Dx 19D, non-uniform grid.

NASA/TM-2000-210225 


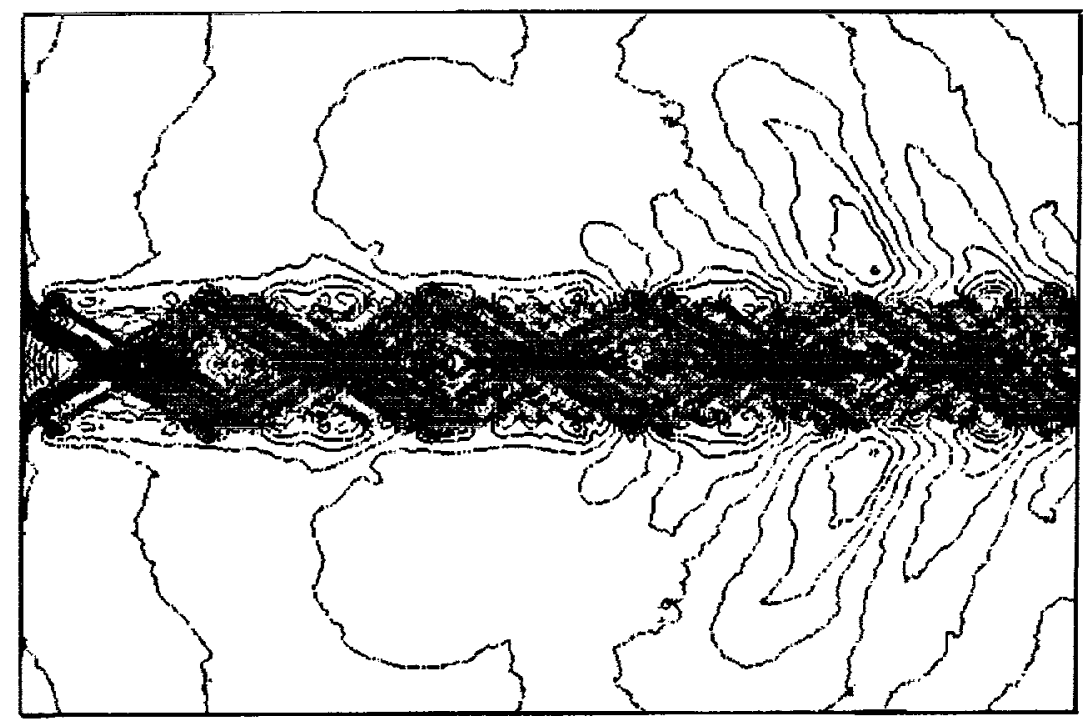

Figure 6: Type II Mach radiation of a supersonic $M_{j}=2.0$ jet at low Strouhal number $\mathrm{St}=0.07$, domain size 8Dx5D, non-uniform grid $630 \times 150$.

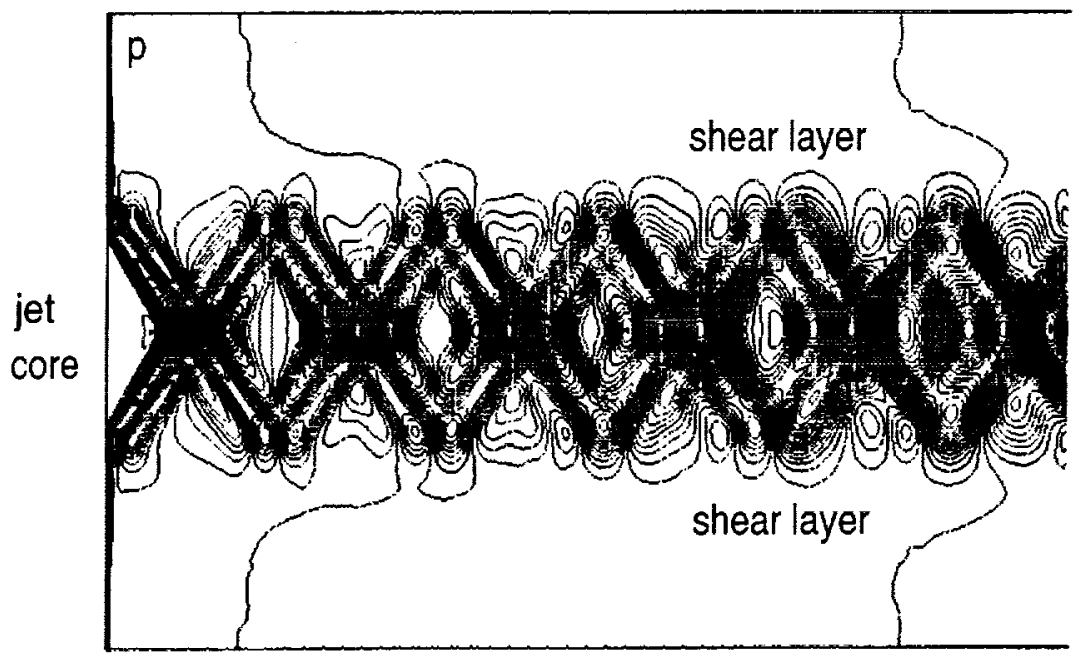

$M \_j=1.2,3 D \times 1 D, 300 \times 100$ grid, St. $=2 . d t=.0015, e e=.00005$; showing cross-hatching Mach wave system.

Figure 7: Mach wave system within a supersonic jet of $M_{j}=1.2$ at high Strouhal number $S t=2.0$, isobars show the cross-hatch pattern. 

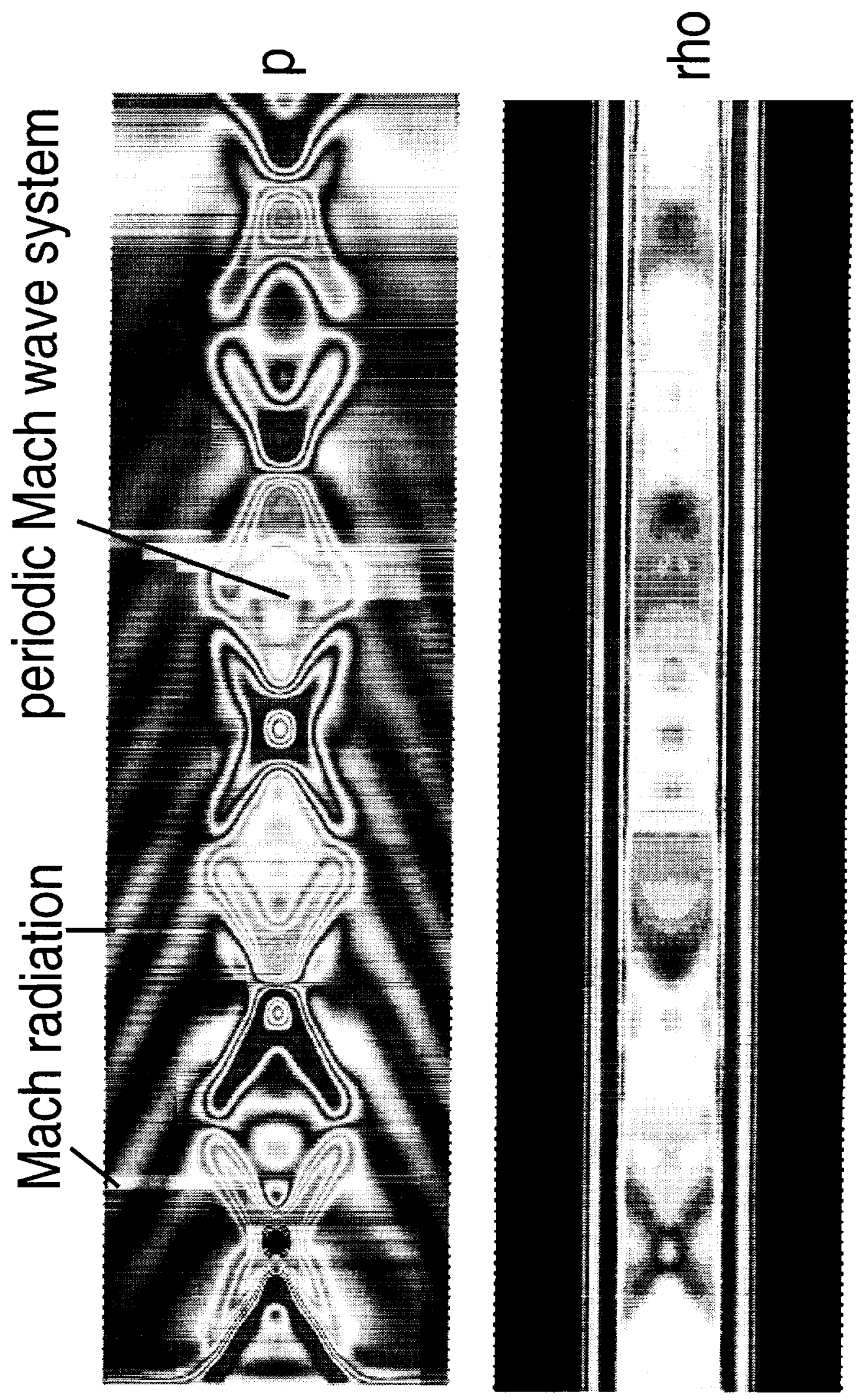

Figure 8: Type III Mach wave system within a supersonic jet of $M_{j}=2$ at high Strouhal number $S t=2.0$, isobars show also weak Mach radiation.

NASA/TM-2000-210225

American Institute of Aeronautics and Astronautics 
Public reporting burden for this collection of information is estimated to average 1 hour per response, including the time for reviewing instructions, searching existing data sources, gathering and maintaining the data needed, and completing and reviewing the collection of information. Send comments regarding this burden estimate or any other aspect of this collection of information, including suggestions for reducing this burden, to Washington Headquarters Services, Directorate for lniformalion Operations and Reports, 1215 Jeflerson Davis Higw, Suite 1204, Arlington, VA 22202-4302, and to the Office of Management and Budget, Paperwork Reduction Project (0704-018B), Washington, DC 20503.

\begin{tabular}{|l|l|r|}
\hline 1. AGENCY USE ONLY (Leave blank) & $\begin{array}{r}\text { 2. REPORT DATE } \\
\text { June } 2000\end{array}$ & $\begin{array}{r}\text { 3. REPORT TYPE AND DATES COVERED } \\
\text { Technical Memorandum }\end{array}$
\end{tabular}

\section{TITLE AND SUBTITLE}

Aeroacoustics Computation for Nearly Fully Expanded Supersonic Jets

Using the CE/SE Method
5. FUNDING NUMBERS

WU $-523-90-43-00$

6. AUTHOR(S)

Ching Y. Loh, Lennart S. Hultgren, Xiao Y. Wang, Sin-Chung Chang, and Philip C.E. Jorgenson

7. PERFORMING ORGANIZATION NAME(S) AND ADDRESS(ES)

National Aeronautics and Space Administration

John H. Glenn Research Center at Lewis Field

Cleveland, Ohio 44135-3191

8. PERFORMING ORGANIZATION REPORT NUMBER

E- 12345

9. SPONSORING/MONITORING AGENCY NAME(S) AND ADDRESS(ES)

10. SPONSORING/MONITORING AGENCY REPORT NUMBER

National Aeronautics and Space Administration

Washington, DC 20546-0001

NASA TM-2000-210225

AIAA-2000-2010

11. SUPPLEMENTARY NOTES

Prepared for the Sixth Aeroacoustics Conference cosponsored by the American Institute of Aeronautics and Astronautics and the Confederation of European Aerospace Societies, Maui, Hawaii, June 12-14, 2000. Ching Y. Loh and Xiao Y. Wang, Taitech Inc., 1430 Oak Court, Suite 301, Beavercreek, Ohio 45430 (work funded under NASA contract NAS3-97186); Lennart S. Hultgren,

Sin-Chung Chang, and Philip C.E. Jorgenson, NASA Glenn Research Center. Responsible person, Philip C.E. Jorgenson, organization code 5880, (216) 433-5386.

12a. DISTRIBUTION/AVAILABILITY STATEMENT

12b. DISTRIBUTION CODE

Unclassified - Unlimited

Subject Categories: 02 and 64

Distribution: Nonstandard

This publication is available from the NASA Center for AeroSpace Information, (301) 621-0390.

13. ABSTRACT (Maximum 200 words)

In this paper, the space-time conservation element solution element (CE/SE) method is tested in the classical axisymmetric jet instability problem, rendering good agreement with the linear theory. The CE/SE method is then applied to numerical simulations of several nearly fully expanded axisymmetric jet flows and their noise fields and qualitative agreement with available experimental and theoretical results is demonstrated.

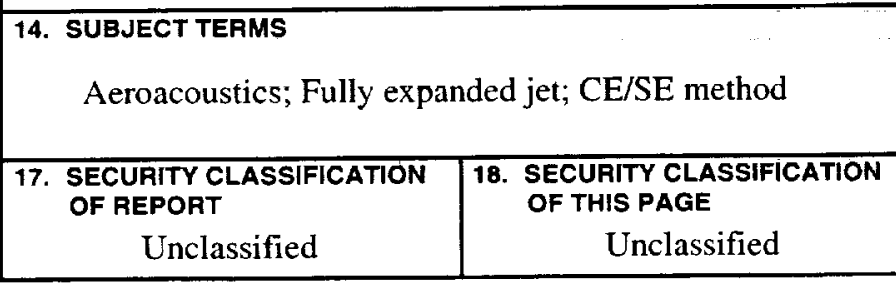

SECURITY CLASSIFICATION
OF ABSTRACT
Unclassified

